\title{
Perceptions of foundation year doctors: barriers to delivering teaching and accessing teaching training
}

\author{
Authors: Helen Parry, Carmen Lau, Natali Vigneswaran, Motahare Yadegarfar and Julia Zhu
}

\section{Aims}

To identify potential barriers which exist for foundation year (FY) doctors in teaching or accessing teaching training.

\section{Methods \\ Clinical teaching fellows designed and delivered two teaching workshops for FY doctors, each consisting of five interactive sessions on a range of topics. FY doctors were invited from three local hospitals; 43 doctors in total attended the teaching workshop. Doctors completed anonymous questionnaires prior to each workshop. Participants were asked to indicate prior experience in teaching or teaching training, as well as if they felt barriers existed in access to either of these. The questionnaire also included items on common perceived barriers to teaching or accessing training in teaching.}

\section{Results}

Forty doctors responded to questions on previous experiences of teaching and teaching training: $36(90 \%)$ doctors had previous teaching experiences and 17 (43\%) doctors had received previous training in teaching. Forty-three doctors answered questions relating to barriers to teaching and attending teaching training; 40 $(93 \%)$ doctors felt barriers existed in delivering teaching. The main barriers included lack of time (85\%, 34 doctors) and limitations in personal knowledge (68\%, 27 doctors). Thirty-four (79\%) doctors felt there were barriers in accessing training in teaching. Reasons identified included lack of time/study leave ( $97 \%, 33$ doctors), as well as a lack of awareness of available courses (85\%, 29 doctors).

\section{Conclusion}

The majority of doctors who attended the workshop felt that barriers did exist in delivering teaching. However, despite this, most participants had managed to get teaching experience. Of note, a significant proportion of doctors felt barriers existed in accessing training and less than half of them had attended formal courses.

Continued development of teaching skills is a key competency of the foundation programme curriculum. Research demonstrates that having attended formal teaching training improves the quality and standard of teaching delivered. Perceived barriers are clearly disproportionately impacting on FY doctors' ability to attend formal teaching courses. This may have a potential impact on the quality of teaching provided, and could affect development of individual teaching standards of FY doctors. This foundation teaching workshop provides an easily accessible formal teaching course. In addition to others available, it provides scope to increase the quality of foundation doctors' teaching.

\section{Conflict of interest statement}

No conflict of interest. 